Babovsky, Hans:

On a Monte Carlo scheme for Smoluchowski's coagulation equation

Zuerst erschienen in: Monte Carlo methods and applications. - Berlin [u.a.] : de Gruyter. - 5 (1999), 1, p. 1-18.

Erstveröffentlichung: 1999

Datum Digitalisierung: $\quad 2009.10 .16$

ISSN (online): $\quad$ 1569-3961

ISSN (print): $\quad$ 0929-9629

DOI: $\quad$ 10.1515/mcma.1999.5.1.1

[Zuletzt gesehen: 2019.08.13]

„Im Rahmen der hochschulweiten Open-Access-Strategie für die Zweitveröffentlichung identifiziert "durch die Universitätsbibliothek IImenau."

"Within the academic Open Access Strategy identified for deposition by IImenau University Library."

„Dieser Beitrag ist mit Zustimmung des Rechteinhabers aufgrund einer (DFGgeförderten) Allianz- bzw. Nationallizenz frei zugänglich."

"This publication is with permission of the rights owner freely accessible due to an Alliance licence and a national licence (funded by the DFG, German Research Foundation) respectively."

\section{DFG}

Nationallizenzen 


\title{
On a Monte Carlo scheme for Smoluchowski's coagulation equation
}

\author{
Hans Babovsky \\ Institute for Mathematics \\ Technical University Ilmenau \\ P. O. Box 100565 \\ D-98684 Ilmenau, Germany \\ E-mail: babovsky@mathematik.tu-ilmenau.de
}

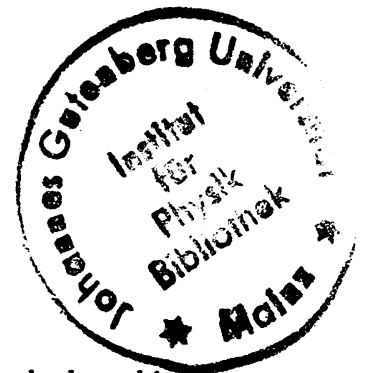

Abstract - We propose a Monte Carlo simulation scheme for the Smoluchowski equation of aerosol dynamics and discuss its numerical efficiency.

Key words: aerosol dynamics, Smoluchowski equation, Monte Carlo simulation

\section{Introduction}

The numerical simulation of aerosol growth is a research field of high current interest. It finds its applications in a variety of engineering contexts ranging from environmental sciences (growing and spreading of air pollutants) to the development of engines (e.g., the modeling of soot formation). The problem of numerically calculating aerosols quickly reaches a complexity which cannot be handled on a computer with reasonable calculational efforts. Think for example of a multicomponent aerosol system diffusively spreading into a gaseous environment with an effective mass distribution ranging from mass one ("elementary") aerosol particle) to several 10 000's.

Due to the complexity of the Smoluchowski equation - the equation governing the spreading and growth of aerosol particles on a mesoscopic level - the main numerical tool are Monte Carlo simulations. The heuristic approach to such stochastic schemes is the intention to reproduce in a reduced particle ensemble a statistics close to that of the real aerosol system. The structure of the Smoluchowski equation - a transport differential operator on the left hand side and a local quadratic particle interaction operator on the right hand side - is closely related to that of the Boltzmann equation, the fundamental equation of rarefied gas dynam- 
ics. Monte Carlo schemes for the Boltzmann equation have been studied intensively during the past decades and are now understood as mathematically rigorous numerical algorithms. (For the correct mathematical setting and the convergence of the simulated solutions to the solutions of the Boltzmann equation, see, e.g. $[3,4,10])$. Moreover, people have learned to modify such schemes in order to increase numerical efficiency. For a survey up to 1995 , see [8]. The intention of the present paper is to investigate in the same spirit stochastic schemes for Smoluchowski's equation.

The paper is organized as follows. In Chapter 2, a modified Monte Carlo scheme for the space homogeneous, discrete Smoluchowski equation is proposed, in which the simulated particles represent unit masses of the aerosol ensemble rather than physical particles. We shortly sketch a convergence proof which is closely related to that in [3]. (Similar convergence results for "standard" Monte Carlo schemes are provided in [6, 5]). Chapter 3 starts with a short outline of a typical two-particle interaction simulation model; we discuss some of its deficiencies. Then numerical results are compared showing a significant reduction of fluctuations for the new method. In Chapter 4, the problem of the appropriate choice of time steps in the case of unbounded interaction operators is addressed.

\section{A Monte Carlo scheme}

\subsection{Modification of a standard scheme}

In the simplest situation (i.e., time homogeneous, without particle dissection), the discrete Smoluchowski equation reads (see, e.g. [11])

$$
\partial_{t} f_{i}=\frac{1}{2} \sum_{k=1}^{i-1} K_{k, i-k} f_{k} f_{i-k}-f_{i} \sum_{l=1}^{\infty} K_{l, i} f_{l}
$$

Here, $f_{i}$ describes the portion (i.e., the relative particle number) of aerosols of size $i$. During the run of time, two particles of sizes $k$ and $l$ may stick together and become one particle of size $k+l$. The rate of merging events is given by the kernel $K_{k, l}$ which is symmetric in $k$ and $l$.

2.1 Examples: Kernels if the quadratic interaction operator which are interesting mainly from an academic point of view are $K_{k, l}^{(1)}=k+l$ and $K_{k, l}^{(2)}=k \cdot l$. The corresponding solutions display a rather different be- 
haviour, e.g. concerning gelation (see the review paper [1] and the literature cited there). Kernels which are more important for practical applications are $K_{k, l}=\left(k^{1 / 3}+l^{1 / 3}\right)^{2}(1 / k+1 / l)^{1 / 2}$ and $K_{k, l}=\left(k^{1 / 3}+\right.$ $\left.l^{1 / 3}\right)\left(k^{-1 / 3}+l^{-1 / 3}\right)$, see [9]. All these kernels represent quadratic operators which are unbounded in the usual $\ell^{q}$-norms.

2.2 Remark: Since particles may stick together, the total number of particles $\sum_{i} f_{i}(t)$ is in general a decreasing quantity, while the whole mass

$$
m(t)=\sum_{i=1}^{\infty} i \cdot f_{i}(t)
$$

is conserved as long as

$$
\sum_{k, l=1}^{\infty} K_{k, l} f_{k} f_{l} \leq C<\infty
$$

Coagulation events may become very complex, e.g. when considering multispecies systems in a spatially inhomogeneous setting (which we will not consider here). Therefore a main tool for the numerical evaluation is the Monte Carlo techique. Here, one tries to imitate the game of the aerosol particles: Calculate probabilities for two-particle interactions (i.e., for two particles sticking together) and simulate these (see [9]). To get a reasonable statistics, the size of the simulated particle system has to be large enough. There are some drawbacks inherent to schemes following this philosophy.

2.3 Remarks: (a) As mentioned in Remark 2.2, the number of particles is decreasing with time. Therefore, one has to take care that from time to time the number of particles has to be artificially increased. [9] describes a way how to do this.

(b) When starting with an ensemble with some maximum size $N_{\max }$, the population of particles with larger sizes stays very poor for small times and thus displays a poor statistics. On the other hand, for the examples 2.1 , the rate of collisions with large particles is high. Thus large statistical fluctuations may be expected.

These deficiencies motivate us to propose the following modification. Rather than simulating the occupation numbers $f_{i}$, we construct a scheme for the occupation masses

$$
g_{i}:=i \cdot f_{i}
$$


As indicated above, the sum $\Sigma_{i} g_{i}(t)=m(t)$ is (formally - because of the restriction (2.3)) a conserved quantity. The equation for $g_{i}$ reads

$$
\begin{aligned}
\partial_{t} g_{i} & =\frac{1}{2} \sum_{k=1}^{i-1}\left(\frac{1}{k}+\frac{1}{i-k}\right) K_{k, i-k} g_{k} g_{i-k}-g_{i} \cdot \sum_{l=1}^{\infty} \frac{1}{l} K_{l, i} g_{l} \\
& =\sum_{k=1}^{i-1} \frac{1}{k} K_{k, i-k} g_{k} g_{i-k}-g_{i} \cdot \sum_{l=1}^{\infty} \frac{1}{l} K_{l, i} g_{l}
\end{aligned}
$$

(for the latter equality we have exploited the symmetry of $K_{k, l}$ ). Standard Euler discretization leads to

$$
g_{i}(t+\Delta t)=\left(1-\Delta t \sum_{l=1}^{\infty} \frac{1}{l} K_{l, i} g_{l}(t)\right) g_{i}(t)+\Delta t \sum_{k=1}^{i-1} \frac{1}{k} K_{k, i-k} g_{k}(t) g_{i-k}(t) \cdot(2.7)
$$

We now construct a Monte Carlo scheme which is closely related to Nanbu's scheme for the Boltzmann equation [7] with the modification proposed in [2]. Without loss of generality we assume that we start at time $t=0$ with a distribution satisfying

$$
\sum_{i=1}^{\infty} g_{i}(0)=1
$$

The scheme is then as follows.

2.4 Monte Carlo algorithm: (a) Initialization of an $\mathrm{N}$-particle system: For $i=1 \ldots N$ choose states $z_{i}(0) \in \mathbb{N}=\{1,2, \ldots\}$ such that

$$
\frac{1}{N} \sharp\left\{i: z_{i}(0)=l\right\} \approx g_{l}(0) .
$$

(b) Propagation step: Suppose an N-particle system $z_{i}(n)$ is given such that

$$
\frac{1}{N} \sharp\left\{i: z_{i}(n)=l\right\} \approx g_{l}(t)
$$

for some $t$.

- For each $i$ choose uniformly distributed random numbers $\pi_{i} \in\{1 \ldots N\}$ and $r_{i} \in[0,1]$.

- Define a time step $\Delta t$ such that

$$
\frac{\Delta t \cdot K_{z_{l}, z_{i}}}{z_{l}(n)} \leq 1, \quad i, l=1 \ldots N .
$$

- Define 


$$
z_{i}(n+1):=\left\{\begin{array}{ll}
z_{i}(n)+z_{\pi_{i}}(n) & \text { if } \\
z_{i}(n) & \text { else. }
\end{array} \quad r_{i} \leq K_{z_{\pi_{i}}, z_{i}} \cdot \Delta t / z_{\pi_{i}}(n)\right.
$$

2.5 Remark: For the choice of the time step it is sufficient to require

$$
\frac{K_{z_{\pi_{i}} z_{i}} \cdot \Delta t}{z_{\pi_{i}}(n)} \leq c, \quad i=1 \ldots N
$$

for a sufficiently small quantity $c$. For the numerical experiments in the following section we choose $c=0.1$.

We are going to show in the following subsection, that under the assumption for the propagation step the updated system is an approximation of $g(t+\Delta t)$, i.e.,

$$
\frac{1}{N} \sharp\left\{i: z_{i}(n+1)=l\right\} \approx g_{l}(t+\Delta t) .
$$

As a first heuristic argument, consider the loss rate of equation (2.7) for a particle in state $i$,

$$
\Delta t \cdot \sum_{k=1}^{\infty} \frac{1}{k} K_{k, i} g_{l}
$$

which because of (2.9) is approximated by

$$
\frac{\Delta t}{N} \sum_{j=1}^{N} \frac{1}{z_{j}} K_{z_{j}, i}
$$

Suppose the $l$-th particle has size $i$, i.e. $z_{l}=i$. Then $\pi_{l}=j$ with probability $1 / \mathrm{N}$. Given $\pi_{l}=j, l$ is removed (i.e., changes size) with probability $K_{z_{j}, i} \cdot \Delta t / z_{j}$. This produces the correct loss rate (2.15).

\subsection{Convergence of the scheme}

2.6 Theorem: Suppose for $N \geq N_{0}$ there are given particle ensembles $\left\{z_{l}^{(N)}(0), l=1 \ldots N\right\}$ approximating $g(0)$ in the sense that

$$
\lim _{N \rightarrow \infty} \frac{1}{N} \sharp\left\{l: z_{l}^{(N)}(0)=i\right\}=g_{i}(0), \quad i \in \mathbb{N} .
$$

Then $\left\{z_{l}^{(N)}(1), l=1 \ldots N\right\}$ as generated by the Monte Carlo scheme 2.4 approximates the solution $g(\Delta t)$ of the discretized equation (2.7), i.e. 


$$
\lim _{N \rightarrow \infty} \frac{1}{N} \sharp\left\{l: z_{l}^{(N)}(1)=i\right\}=g_{i}(\Delta t), \quad i \in \mathbb{N} .
$$

The proof follows the lines of that for the modified Nanbu scheme [3].

Proof: Fix some number $i \in \mathbb{N}$ and denote

$$
\chi_{i}^{(N)}(l):= \begin{cases}1 & \text { if } z_{l}^{(N)}(1)=i \\ 0 & \text { else } .\end{cases}
$$

Then

$$
\sharp\left\{l: z_{l}^{(N)}(1)=i\right\}=\sum_{l=1}^{N} \chi_{i}^{(N)}(l)=\sum_{j \in \mathbb{N}}\left(\sum_{l: z_{l}^{(N)}(0)=j} \chi_{i}^{(N)}(l)\right) .
$$

The terms $\sum_{l: z_{l}^{(N)}(0)=j} \chi_{i}^{(N)}(l)$ are sums of independent equally distributed random numbers. The expectations may be obtained from the formula of conditional expectations,

$$
\mathcal{E} \chi_{i}^{(N)}(l)=\sum_{k \in \mathbb{N}} P\left(z_{l}^{(N)}(1)=i \mid z_{l}^{(N)}(0)=j, z_{\pi_{l}}(0)=k\right) \cdot P\left(z_{\pi_{l}}(0)=k\right)(2.21)
$$

From the assumptions follows

$$
P\left(z_{\pi_{l}}(0)=k\right)=\frac{1}{N} \sharp\left\{m: z_{m}^{(N)}(0)=k\right\} .
$$

From the transition rates (2.12) of the Monte Carlo scheme we find

$$
\begin{aligned}
P\left(z_{l}^{(N)}(1)=i \mid z_{l}^{(N)}(0)\right. & \left.=j, z_{\pi_{l}}^{(N)}(0)=k\right) \\
& =\left\{\begin{array}{lll}
1-\Delta t \cdot K_{k, j} / k & \text { if } j=i \\
\Delta t \cdot K_{k, j} / k & \text { if } j+k=i \\
0 & \text { else }
\end{array}\right.
\end{aligned}
$$

yielding for $N \rightarrow \infty$

$$
\mathcal{E} \chi_{i}^{(N)}(l) \longrightarrow\left\{\begin{array}{lll}
\sum_{k \in \mathbb{N}}\left(1-\Delta t \cdot K_{k, j} / k\right) g_{k}(0) & \text { if } & i=j \\
\Delta t \cdot K_{i-j, j} g_{i-j} /(i-j)(0) & \text { if } & i>j \\
0 & \text { else }
\end{array}\right.
$$




$$
\frac{1}{N} \sharp\left\{m: z_{m}^{(N)}(0)=k\right\} \longrightarrow g_{k}(0),
$$

the strong law of large numbers yields

$$
\begin{aligned}
& \lim _{N \rightarrow \infty} \frac{1}{D_{l j}^{N}} \sum_{l: z_{l}^{(N)}(0)=j} \chi_{i}^{(N)}(l) \\
& =\lim _{N \rightarrow \infty} \frac{1}{D_{l j}^{N}} \sum_{l: z_{l}^{(N)}(0)=j} \sum_{k \in \mathbb{N}} P\left(z_{l}^{(N)}(1)=i \mid z_{l}^{(N)}(0)=j, z_{\pi_{l}}^{(N)}(0)=k\right) g_{k}(0) \\
& =\sum_{k \in \mathbb{N}} P\left(z_{l}^{(N)}(1)=i \mid z_{l}^{(N)}(0)=j, z_{\pi_{l}}^{(N)}(0)=k\right) g_{k}(0)
\end{aligned}
$$

where $D_{l j}^{N}=\sharp\left\{l: z_{l}^{(N)}(0)=j\right\}$. Inserting this into

$$
\frac{1}{N} \sum_{l=1}^{N} \chi_{i}^{(N)}(l)=\sum_{j \in \mathbb{N}} \frac{D_{l j}^{N}}{N}\left(\frac{1}{D_{l j}^{N}} \sum_{l: z_{l}^{(N)}(0)=j} \chi_{i}^{(N)}(l)\right)
$$

the law of large numbers yields

$$
\frac{\sharp\left\{l: z_{l}^{(N)}(1)=i\right\}}{N} \longrightarrow \sum_{j \in \mathbb{N}} g_{j}(0)\left(\lim _{N \rightarrow \infty} \mathcal{E}\left(\chi_{i}^{(N)}(l) \mid z_{l}=j\right)\right) .
$$

Inserting the values (2.24) leads to the desired result.

2.7 Remark: (a) Let $\Delta t_{n}$ be the time step of the $n$-th iteration of the Monte Carlo scheme. Then by induction it follows that $\left\{z_{l}^{(N)}(n), l=\right.$ $1 \ldots N\}$ approximates the discretized solution $g\left(\Delta t_{1}+\ldots+\Delta t_{n}\right)$ (i.e., the solution of (2.7) solved with subsequent time steps $\Delta t_{1}, \ldots, \Delta t_{n}$ ) for all $n \in \mathbb{N}$.

\section{Numerical results}

\subsection{Standard Monte Carlo schemes}

Monte Carlo schemes based on heuristic arguments are intended to imitate the two-particle dynamics of a real aerosol ensemble. This means that two particles which interact merge into one larger particle. This is in contrast to the dynamics introduced in section 2 . There, the results after one time step form $N$ stochastically independent events, i.e., there is no simultaneous change of particles and their collision partners, and the number of "particles" (i.e., of unit masses) remains equal to $N$. 
To simulate the two-particle interaction model, the propagation step of the Monte Carlo scheme 2.4 has to be modified as follows.

- Rather than choosing $N$ independent "collision partners" $\pi_{i}$, choose a random permutation $P$ of $\{1 \ldots N\}$ and consider the pairs $(P(2 i-$ 1), $P(2 i)$ ), $i=1 \ldots N / 2$ as "collision pairs". (If $N$ is odd, the $N$-th particle remains "without partner").

- With probability

$$
p_{i}=\Delta t \cdot K_{z_{P(2 i-1)}, z_{P(2 i)}} \cdot \frac{N_{a c t}}{N_{r e f}} \leq 1
$$

change the mass of the $P(2 i-1)$-th particle from $z_{P(2 i-1)}$ into $z_{P(2 i-1)}+$ $z_{P(2 i)}$ and drop the $P(2 i)$-th particle from the list. Here, $N_{\text {ref }}$ is a reference particle number representing the state $\sum_{n \in \mathbb{N}} f_{i}=1 . N_{\text {act }}$ is the actual particle number.

Considering the different collision models, there are pros and cons for both alternatives. Standard random schemes as described above (we refer to these in the following as Model 2,) are more intuitive, since particles represent physical particles, and their interaction is based on a two-particle collision model (i.e., two particles change their state simultaneously and in dependence of one another). Contrary to this, all particles of the alternative model of section 2 (called Model 1) change their state stochastically independent of the other particles. It has to be tested whether this introduces artificial fluctuations into the scheme. (However, this does not affect any conserved quantity ${ }^{1}$ ). On the other hand, merging of two particles into one means that the total number of particles is decreasing thus deteriorating the statistics of the whole particle ensemble.

Figure 1 shows the development of particle numbers for a scheme for the kernel $K_{k, l}=k+l$. To ensure a reasonable statistics, the total number of particles has to be artificially increased after a couple of time steps. A way of how to do this has been indicated in [9]. To obtain the results presented below, we start with a system of $N_{\text {ref }}$ particles and double the number of particles whenever the actual number decreases below the value of $2 N_{\text {ref }} / 3$. (Notice that in order to maintain the correct collision frequency, the probability (3.1) has to be halved whenever the particle number is doubled).

\footnotetext{
${ }^{1}$ It was the fluctuation of conserved quantities which initially called into doubt the validity of Nanbu's scheme. A modification into a two-particle interaction model with strict conservation was proposed in [3, Sec. 7]. However, because of the asymmetric kernel $(1 / k) K_{k, l}$, this modification seems not to be applicable in our case.
} 


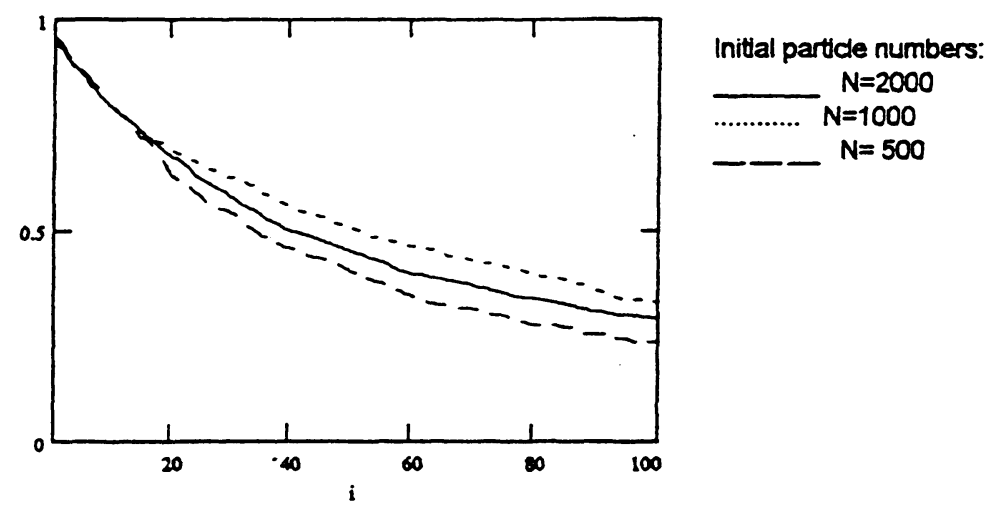

Figure 1. Decrease of particle numbers, model 2

Doubling is performed by producing a copy of the actual particle system. Of course, this procedure does not diminish the fluctuations which have built up so far. In contrast to the two-particle interaction scheme, the modified scheme of Section 2 works all the time with a fixed particle number, each "particle" representing one unit mass rather than one aerosol particle.

\subsection{Comparison of numerical results}

When including a variety of physical effects into the Monte Carlo simulation like multicomponent systems with chemical reactions in a spatially varying environment, the numerical task becomes very time (and computer memory) consuming. Thus an efficient scheme has to yield reasonable results even for modest particle numbers. Therefore our main concern is now the comparison of numerical results for the two models introduced above. For the collision kernel $K_{k, l}=k+l$ we have performed simulation runs for particle numbers ranging from 2000 down to 500 . The results are demonstrated in Figs. 4 to 6 in the appendix. In all cases, the initial configuration is that of a system consisting of mass-one particles only. The first bar in each figure describes the total amount of particles with 
mass one after different time intervals. The second bar indicates the total mass of all particles with masses between two and ten. The $k$-th bar, $k=3 \ldots 11$, concerns the total mass of particles from size $10 \cdot(k-2)+1$ up to size $10 \cdot(k-1)$. We notice that the qualitative behaviour is in quite good agreement for both models. However, the model 2 reveals considerably larger fluctuations, especially for large time intervals and for small particle ensembles. In contrary, model one exhibits reasonably smooth distributions. When changing from the 2000-particle system to the 500-particle system, there is essentially no change of the results. This model allows to decrease the particle numbers even more.

Let us also compare the evolution of certain moments of the particle distributions. For a given solution $f$ of the Smoluchowski equation denote by

$$
\Phi(s, t):=\sum_{i \leq s} f_{i}(t)
$$

the (discrete) particle distribution at time $\mathrm{t}$ and by $\Phi(d s, t)$ the corresponding Stieltjes integral with respect to $s$. If $g$ is the solution of the Smoluchowski equation with the modification of Section 2, then the total mass

$$
M_{1}(t)=\int_{\mathbb{R}_{+}} s \Phi(d s, t)=\sum_{i=1}^{\infty} g_{i}(t)
$$

is the only conserved quantity in both schemes. Since the states $z_{i}(t)$ of the particles are increasing with time, the second moment

$$
M_{2}(t)=\int_{\mathbb{R}_{+}} s^{2} \Phi(d s, t)=\sum_{i=1}^{\infty} i g_{i}(t)
$$

is a strictly increasing function. The second derivative of $M_{2}$ may serve as a measure for the fluctuations inherent to the schemes. The discrete analog of the second derivative is

$$
\psi_{n}=\psi(n \Delta t):=\frac{1}{\Delta t^{2}}\left(\left(M_{1}((n+1) \Delta t)-2 M_{1}(n \Delta t)+M_{1}((n-1) \Delta t)\right)\right.
$$

In Figure 2, for $\Delta t=0.01$ the moments $\psi_{n}, n=1 \ldots 100$, are plotted for both models and for $N=2000$ and $N=500$. As one may notice, Model 1 reveals much reduced fluctuations when compared to Model 2 - in particular for the small particle system with initially $N=500$ 
particles. This is in agreement with what we have observed in Figures 4, 5 and 6 . Since $M_{2}$ is monotonely increasing, we expect large fluctuations to induce strongly increased values of this function. Inspection of the numerical results yields at $t=1$ (i.e., for $N=100$ ) and for $N=500$ the values $M_{2}(t)=6.80$ for Model 1 and $M_{2}(t)=15.93$ for Model 2 .

We conclude that at least for the 500-particle system, Model 2 yields solutions which reveal large errors connected with moments of the distribution.

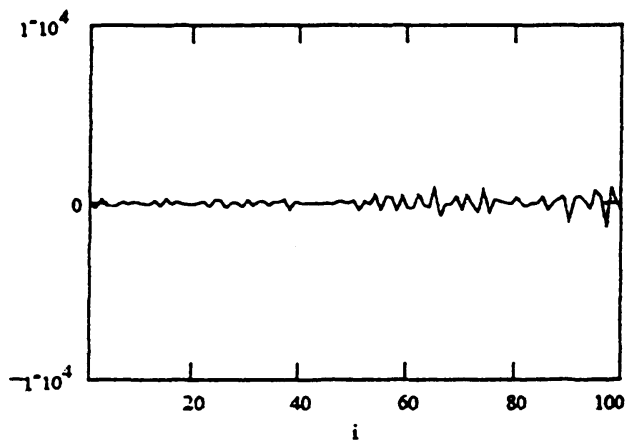

1. Modell $1, N=2000$

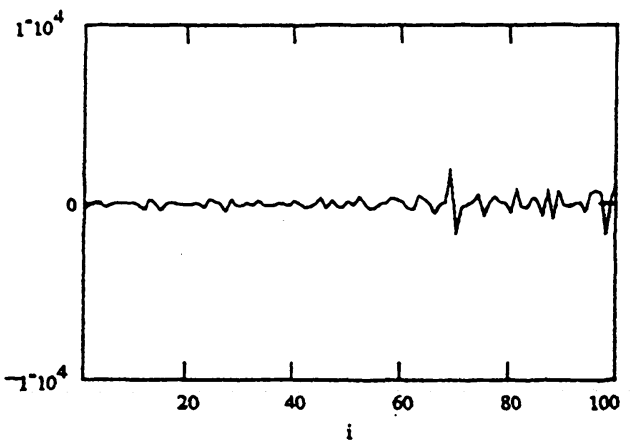

2. Modell $1, N=500$

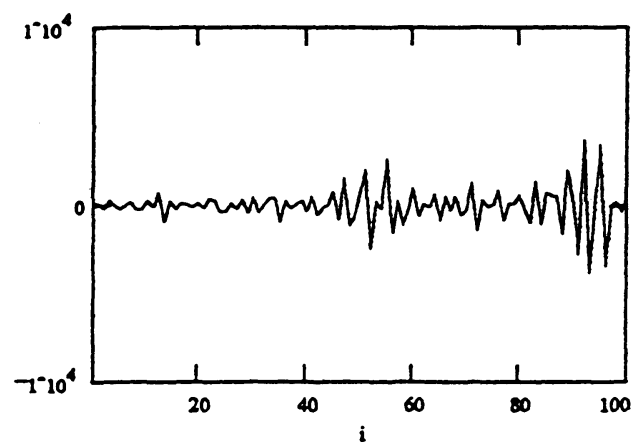

1. Modell 2, $N=2000$

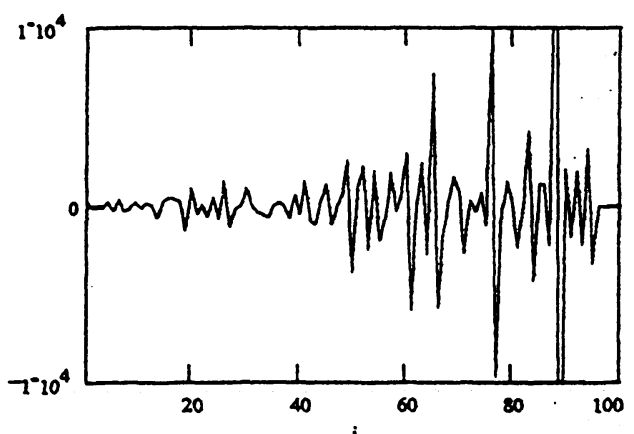

2. Modell 2, $N=500$

Figure 2. Fluctuations of the second moment

\section{The choice of time steps}

Let us finally discuss shortly the problem of appropriate time steps for the scheme. As for all numerical time discretizations, small time steps are desirable for reasons of accuracy, while large time steps reduce calculation 
times on a computer. What makes the problem even harder is the fact that in most cases of interest the quadratic particle interaction operator is unbounded (see Examples 2.1). In the case of the Boltzmann equation, unbounded collision operators are usually coped with by truncating the collision kernel (see, e.g. the remarks in [3]). For aerosol dynamics, the situation is more involved due to the fact that the portion of particles affected by large collision frequencies (i.e., large particles) is increasing with time (see Lemmata 4.8 and 4.9). In this section we first investigate particle growth for one particular interaction model and finally present an example for which the "freezing" of particle sizes (and thus artificially truncating the interaction operator) leads to numerical subsolutions of the initial value problem.

4.8 Lemma: Suppose $K_{k, l}>0$ for all $k, l \in \mathbb{N}=\{1,2, \ldots\}$. Suppose further that there exists a (classical) nonnegative solution $\mathbf{f}=\left(f_{i}\right)_{i \in \mathbb{N}}$ to Smoluchowski's equation $(2.1)$ in some interval $\left[0, t_{0}\right]$, for which the functions

$$
t \longrightarrow \sum_{k \in \mathbb{N}} K_{k, i} f_{k}(t)
$$

are integrable, and $f_{i_{0}}>0$ for some $i_{0} \in \mathbb{N}$. Then for all $t \in\left(0, t_{0}\right]$, the support $\operatorname{supp}(f(t))$ of $f(t)$ defined as

$$
\operatorname{supp}(\mathbf{f}(t))=\left\{i \in \mathbb{N}: f_{i}(t) \neq 0\right\}
$$

is unbounded.

Proof: For $i \in \mathbb{N}$ denote

$$
\phi_{i}(t):=\int_{0}^{t} \sum_{k \in \mathbb{N}} K_{k, i} f_{k}(s) d s .
$$

From the assumptions follows that $\phi_{i}(t)<\infty$. From the integral version of $f_{i}$,

$$
\begin{aligned}
f_{i}(t) & =f_{i}(0) \exp \left(-\phi_{i}(t)\right) \\
& +\int_{0}^{t} \sum_{k=1}^{i-1} K_{k, i-k} f_{k}(s) f_{i-k}(s) \exp \left(-\left(\phi_{i}(t)-\phi_{i}(s)\right)\right) d s
\end{aligned}
$$

we find

$$
f_{i_{0}}(t) \geq f_{i_{0}}(0) \exp \left(-\phi_{i_{0}}(t)\right)>0
$$

and by induction 


$$
f_{(n+1) i_{0}}(t) \geq \exp \left(-\phi_{n i_{0}}(t)\right) \int_{0}^{t} K_{n i_{0}, i_{0}} f_{n i_{0}}(s) f_{i_{0}}(s) d s>0
$$

Since particle masses are monotoneously increasing, large particles contribute more and more to collision events, while small particles contribute negligibly. Precisely, we have

4.9 Lemma: Let $\mathbf{g}=\left(g_{i}\right)_{i \in \mathbb{N}}$ be a nonnegative solution of equation (2.6) satisfying $\sum_{i \in \mathbb{N}} g_{i}(t)=1$. If $(1 / k) K_{k, l} \geq \kappa^{(0)}$ for all $k, l$, then the total mass

$$
\gamma_{N}(t):=\sum_{i=1}^{N} g_{i}(t)
$$

of all particles of size not larger than $N$ decays as follows,

$$
\gamma_{N}(t) \leq \frac{\gamma_{N}(0) \exp \left(-\kappa^{(0)} t\right)}{1-\gamma_{N}(0)\left(1-\exp \left(-\kappa^{(0)} t\right)\right.}
$$

Proof: Summing over all $g_{i}, i=1 \ldots N$, we find

$$
\begin{aligned}
\partial \gamma_{N} & =\sum_{i, j \leq N: i+j>N} g_{i} \cdot \frac{1}{j} K_{j, i} g_{j} \\
& \leq-\kappa^{(0)} \cdot \sum_{i=1}^{N} g_{i} \cdot \sum_{j=N+1}^{\infty} g_{j}=-\kappa^{(0)} \gamma_{N}\left(1-\gamma_{N}\right) .
\end{aligned}
$$

The estimate follows by Gronwall's inequality from solving the equation for the upper solution $u(t)$,

$$
\partial_{t} u=-\kappa^{(0)} u(1-u) . \quad \square .
$$

Consider now the Euler discretization as proposed in equation (2.7). If the collision kernel $(1 / l) K_{l, i}$ is monotoneously increasing in both components, and if it is for fixed $l$ unbounded in $i$, then the system of differential equations for $g_{i}$ contains "stiff" components (i.e., those components which are related to large particle sizes) in the sense that there is no choice for the time step $\Delta t$, for which the loss term of the time discretization is bounded by some term $c \cdot g_{i}$ with $|c|<1$. In particular, conservation of nonnegativity is not guaranteed. To avoid instabilities of the scheme, one way out might be to truncate particle interactions of large particles. However, as we learn from Lemma 4.9, large particles 
become dominating with increasing time. An alternative which is applicable to the scheme presented in Section 2 is as follows. We choose a limit value $N_{0}$ and "freeze" particles whose mass has crossed this level from below. However, these particles are available as "collision partners" for smaller particles. We have simulated test runs for the interaction kernel $K_{k, l}=k+l$. The results at time $t=3$ with particles "frozen" at $N_{0}=100$ (lower curve), 1000 and 10000 (upper curve) are shown in Figure 3. There is numerical evidence that the distribution functions $\Phi^{\left(N_{0}\right)}(t)$ are monotonically increasing with $N_{0}$ - at least for $s \leq 100$. If monotonicity can be strictly established (which goes beyond the aim of the present paper) then this modification leads to the calculation of subsolutions with fixed lower bounds for the time step, and the solution of the original problem is obtained in the limit $N_{0} \rightarrow \infty$.

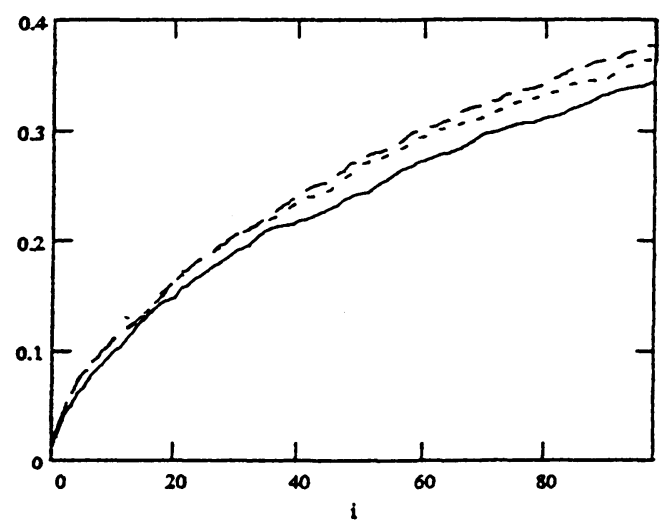

Figure 3. Particle distribution at time $t=3$ for "frozen" systems.

\section{References}

[1] D. J. Aldous. Deterministic and Stochastic Models for Coalescence (Aggregation, Coagulation): a review of the mean-field theory for probabilists. Preprint, University of California, Berkeley, 1997.

[2] H. Babovsky. On a simulation scheme for the Boltzmann equation. Math. Meth. in the Appl. Sci., 8:223-233, 1986.

[3] H. Babovsky. Convergence proof for Nanbu's Boltzmann simulation scheme. Europ. J. Mech. B/Fluids, 1:41-55, 1989. 
[4] H. Babovsky and R. Illner. A convergence proof for Nanbu's simulation method for the full Boltzmann equation. SIAM J. Numer. Anal., 26:45-65, 1989.

[5] A. Kolodko, K. Sabelfeld and W. Wagner. A stochastic method for solving Smoluchowski's coagulation equation. Preprint No. 423, WIAS, Berlin, 1998.

[6] A. Kolodko and W. Wagner. Convergence of a Nanbu type method for the Smoluchowski equation. Monte Carlo Methods and Applications, 3:255-273, 1997.

[7] K. Nanbu. Direct simulation scheme derived from the Boltzmann equation. J. Phys. Soc. Japan, 49:2042, 1980.

[8] H. Neunzert and J. Struckmeier. Particle methods for the Boltzmann equation. Acta Numerica, 417-457, 1995.

[9] K. K. Sabelfeld, S. V. Rogasinsky, A. A. Kolodko and A. I. Levykin. Stochastic algorithms for solving Smolouchovsky coagulation equation and applications to aerosol growth simulation. Monte Carlo Methods and Appl., 2:41-87, 1996.

[10] W. Wagner. A convergence proof for Bird's direct simulation Monte Carlo method for the Boltzmann equation. J. Statist. Phys., 66:1011-1044, 1992.

[11] M. M. R. Williams and S. K. Loyalka. Aerosol Science. Theory and Practice. Pergamon, New York, 1991. 


\section{Appendix}

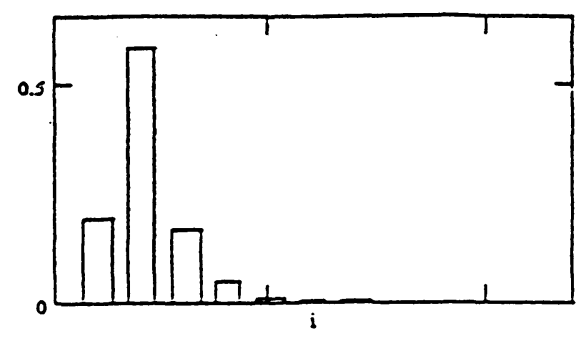

1.1 Modell $1, \mathrm{~N}=2000, t=1.00$

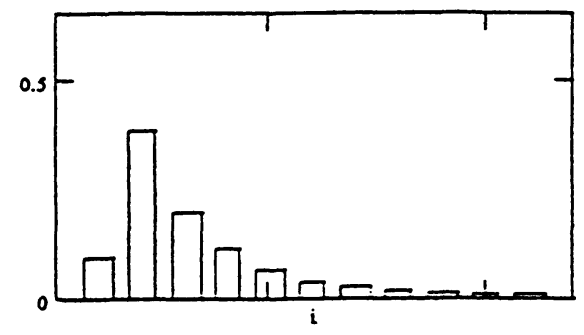

1.2 Modell $1, \mathrm{~N}=2000, \mathrm{t}=1.50$

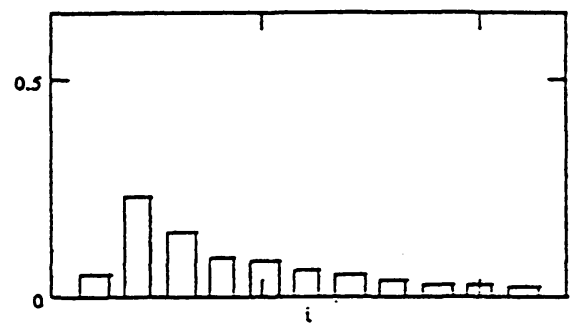

1.3 Modell 1, $N=2000, t=200$

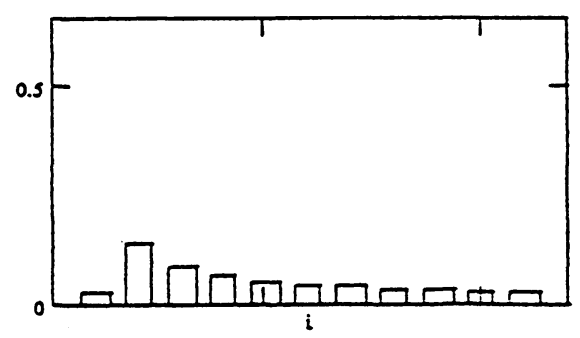

1.4 Model $1, N=2000, t=2.50$

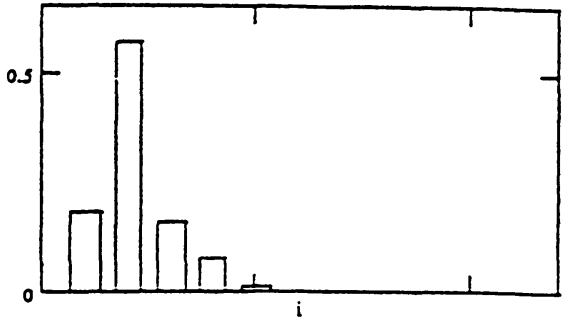

1.1 Modell 2, $N=2000, t=1 . C 0$

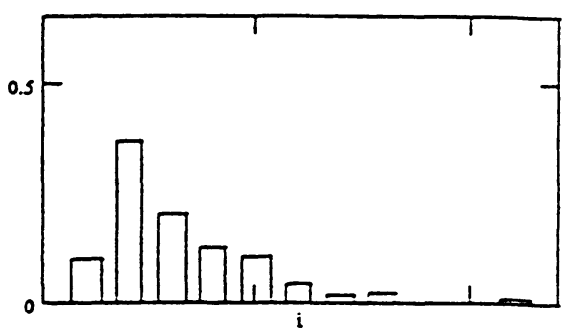

1.2 Modell 2, $\mathrm{N}=2000, \mathrm{t}=1.50$

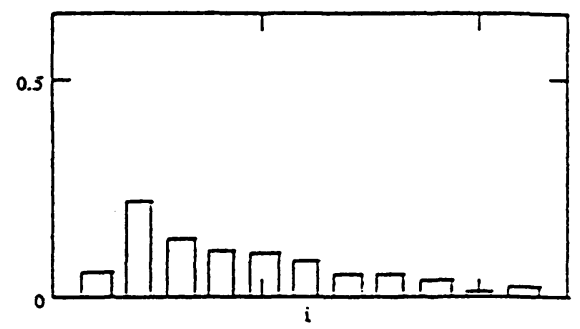

1.3 Modeil 2, $\mathrm{N}=2000, t=200$

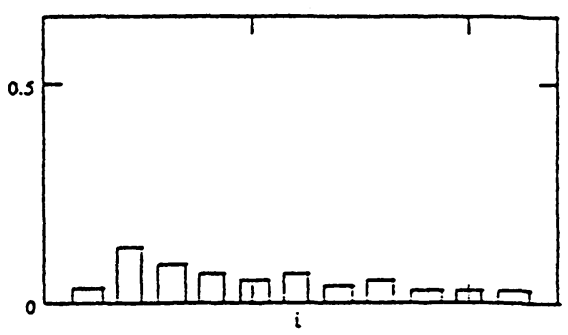

1.4 Modell 2, $N=2000, t=2.50$

Figure 4: Size distributions for a 2000-particle Monte Carlo ensemble 


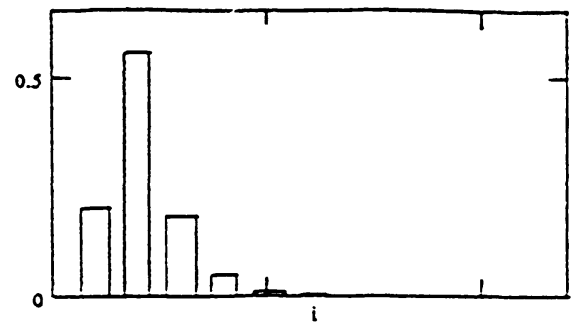

21 Modell $1, N=1000, t=1.00$

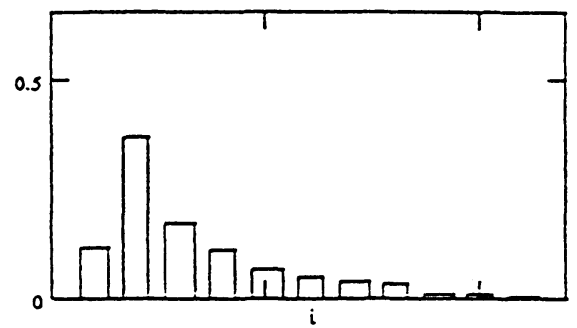

2.2 : Wodell I, $\mathrm{N}=1000, t=1.50$

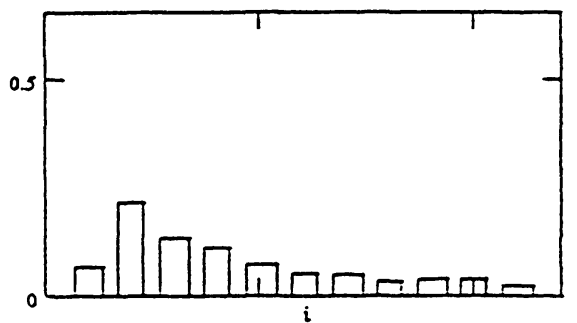

23 Modell $1, N=1000, t=200$

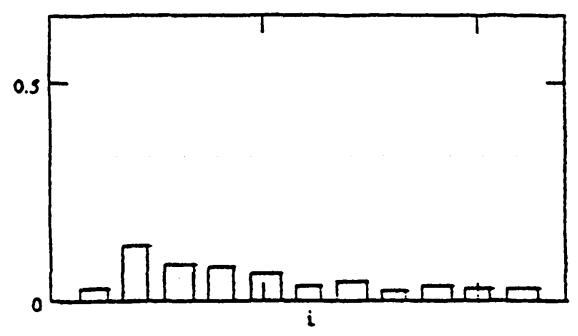

2.4 Modell $1, N=1000, t=2.50$

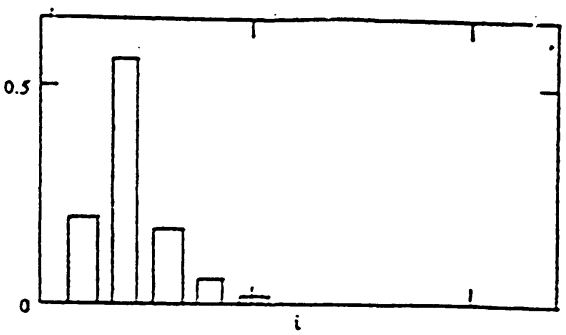

2.1 Modeil 2, $\mathrm{N}=1000, t=1 . \infty 0$

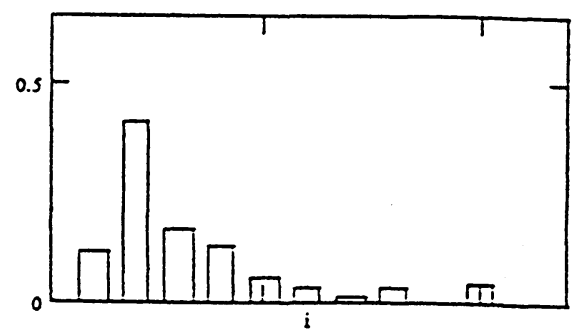

2.2 Modell 2, $\mathrm{N}=1000, t=1.50$

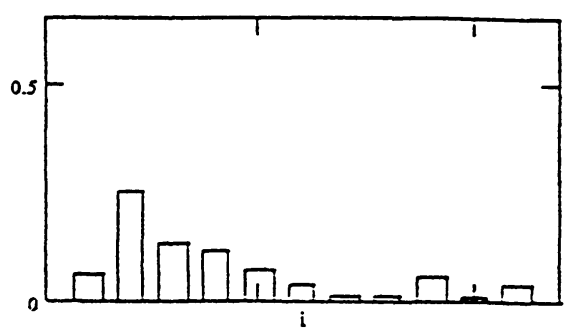

2.3 Wodell 2, Ni=1000, $=2 \infty$

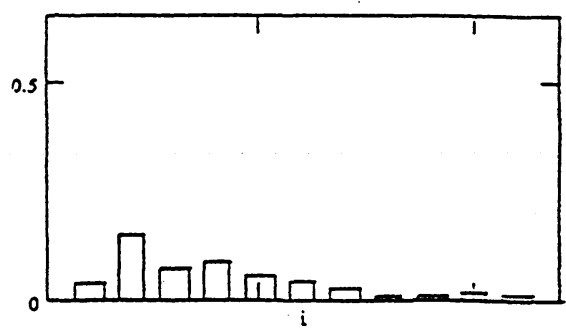

24 MLCell 2, $N=1000, t=2.50$

Figure 5: Size distributions for a 1000-particle Monte Carlo ensemble 


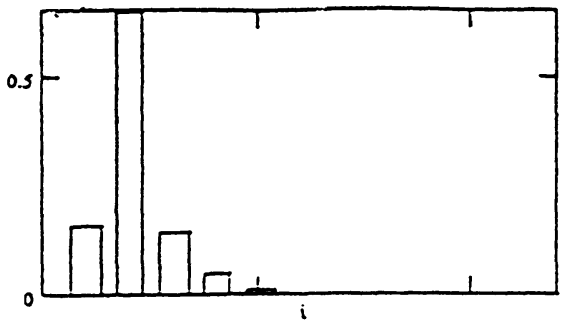

3.1 Madel $1, \mathrm{~N}=500, t=1.00$

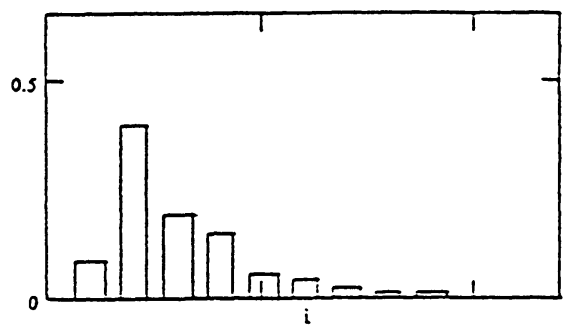

3.2 Modell $1, N=500, F=1.50$

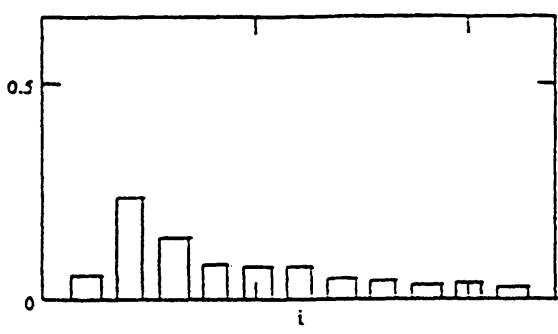

3.3 Modell 1, $N=500, t=2.00$

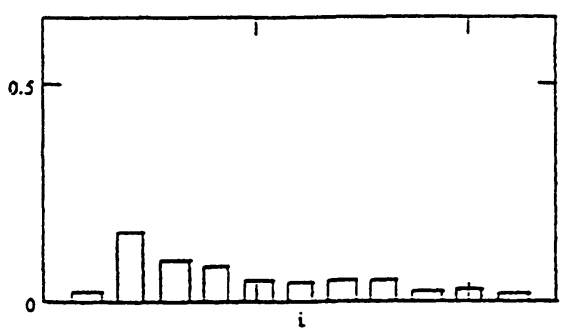

3.4 Mrodeil $1, \mathrm{~N} \rightarrow \mathrm{CO}, \mathrm{F}=2.50$

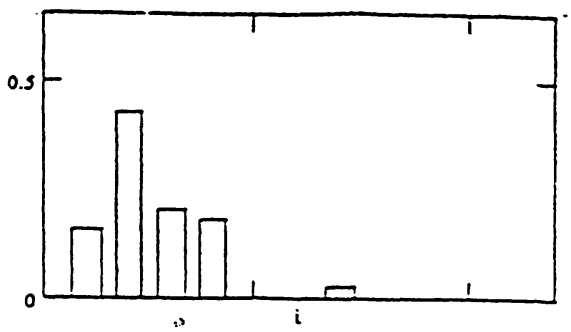

3.1. Modeil 2, $\mathrm{N}=500, t=1.00$

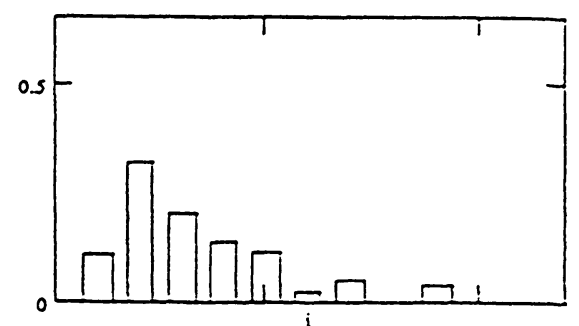

3.2. Mrodell $2, \mathrm{~N}=500, t=1.50$

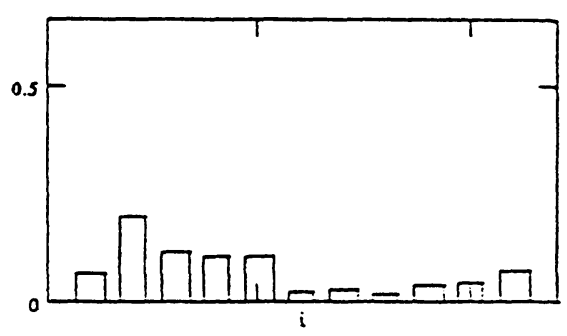

3.3 Micdell 2, $\mathrm{N}=500, t=200$

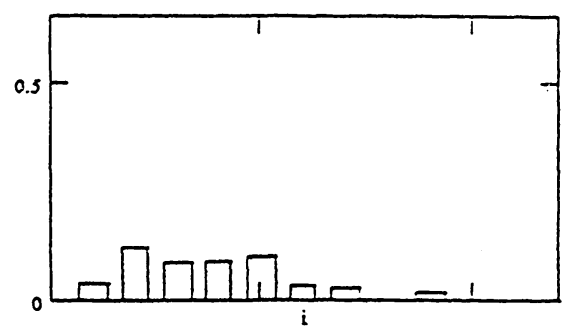

3.4 Modell 2, $N=500, t=2.50$

Figure 6: Size distributions for a 500-particle Monte Carlo ensemble 